\title{
PERCEPCIÓN LATINOAMERICANA DE CAMBIO CLIMÁTICO: METODOLOGÍAS, HERRAMIENTAS Y ESTRATEGIAS DE ADAPTACIÓN EN COMUNIDADES LOCALES. UNA REVISIÓN
}

\section{LATIN AMERICAN PERCEPTIONS OF CLIMATE CHANGE: METHODOLOGIES, TOOLS AND ADAPTATION STRATEGIES IN LOCAL COMMUNITIES. A REVIEW}

\author{
Emma Lucía Forero ${ }^{1}$, Yolanda Teresa Hernández², Carlos Alfonso Zafra ${ }^{3}$
}

\begin{abstract}
${ }^{1}$ Magíster en Desarrollo Sustentable y Gestión Ambiental, Facultad de Medio Ambiente y Recursos Naturales, Universidad Distrital Francisco José de Caldas, Av. Circunvalar Venado de Oro, Bogotá, Colombia, e-mail: emmaluforero@hotmail.com; ${ }^{2}$ Doctora en Geografía, Docente investigadora, Universidad Distrital Francisco José de Caldas, Facultad de Medio Ambiente y Recursos Naturales, Av. Circunvalar Venado de Oro, Bogotá, Colombia, e-mail: ythernandezp@udistrital.edu.co; ${ }^{3}$ Doctor en Ingeniería Ambiental, Profesor Asociado, Universidad Distrital Francisco José de Caldas, Facultad de Medio Ambiente y Recursos Naturales, Av. Circunvalar Venado de Oro, Bogotá, Colombia e-mail: czafra@udistrital.edu.co.
\end{abstract}

Rev. U.D.C.A Act. \& Div. Cient. 17(1): 73-85, Enero-Junio, 2014

\section{RESUMEN}

El estudio del conocimiento, creencias y prácticas que tienen las comunidades con respecto al cambio climático contribuye a llenar vacíos de información científica y prepara el camino para el diseño de medidas de adaptación. En este artículo, se presenta una revisión bibliográfica acerca de las investigaciones de percepción del cambio climático que se han realizado en Latinoamérica, durante los últimos quince años (19972012), en comunidades locales campesinas e indígenas. Al respecto, se identificaron y se analizaron las metodologías, las herramientas y las estrategias de adaptación, reportadas en los estudios de percepción. Los resultados muestran la existencia de tres enfoques metodológicos para abordar los estudios de percepción: (i) cuantitativo, (ii) cualitativo y (iii) mixto. Además, la investigación bibliográfica evidencia que las tres herramientas principales utilizadas en los estudios de percepción son: (i) entrevista semi-estructurada (76,9\%); (ii) encuesta $(42,3 \%)$ y, (iii) observación en campo $(30,8 \%)$. Las herramientas identificadas son utilizadas, principalmente, en estudios de percepción en comunidades campesinas $(65,4 \%)$, antes que en comunidades indígenas $(34,6 \%)$. Por otro lado, se identificaron las siguientes percepciones relevantes: (i) variabilidad climática $(94,1 \%$ ), (ii) incremento de temperatura $(91,2 \%)$, (iii) cambio en la precipitación $(85,3 \%)$ y, (iv) cambio en los hábitos culturales (55,9\%). Los resultados sugieren que la percepción dominante es que existe un cambio asociado, principalmente, con el factor climático; lo anterior, representado por un 90,2\%, de los documentos detectados. Finalmente, las estrategias de adaptación al cambio climático en comunidades locales latinoamericanas, se pueden agrupar en tres categorías: (i) agropecuaria (57,6\%), (ii) comunitaria $(48,1 \%)$, e (iii) hídrica $(40,4 \%)$.

Palabras clave: Percepción climática, cambio climático, estrategias de adaptación, herramientas metodológicas.

\section{SUMMARY}

The study of the knowledge, beliefs and practices that the local communities have respect to climate change contributes to filling voids in the scientific information and prepares the way for designing adaptation actions. This article presents a literature review about the investigations of climate change perception that have been made in Latin America in the last fifteen years (1997-2012) in rural and indigenous communities. In this sense, it was identified and analyzed the methodologies, tools and adaptation strategies reported in perception studies. The results showed that there are three methodological approaches in the perception studies of climate change: (i) quantitative, (ii) qualitative, and (iii) mixed. In addition, the bibliographical research shows that the three main tools used in perception studies are: (i) semi-structured interview (76.9\%), (ii) poll (42.3\%), and (iii) field observation 
(30.8\%). In this regard, the tools identified are mainly used in the perception studies in farming communities (65.4\%) before than in indigenous communities (34.6\%). Furthermore, it was identified the following important perceptions: (i) climate variability (94.1\%), (ii) temperature increase $(91.2 \%)$, (iii) changes in precipitation (85.3\%), and (iv) change in cultural habits (55.9\%). Results suggest that the dominant perception is that there is a change was mainly due to the climatic factor; above, represented by $90.2 \%$ of the documents identified. Finally, the strategies for adaptation to climate change in Latin American local communities can be grouped into three categories: (i) agriculture (57.6\%), (ii) community (48.1\%), and (iii) water (40.4\%).

Key words: Climatic perception, climate change, adaptation strategies, methodological tools.

\section{INTRODUCCIÓN}

Cada cultura tiene sus propias concepciones y percepciones sobre la naturaleza y sus territorios, al igual que sus propias interpretaciones sobre la historia de los cambios ambientales y climáticos, que han ocurrido (Heyd, 2010). Las investigaciones sobre los diversos niveles de conocimiento y percepciones del cambio climático han venido en aumento en los últimos diez años, alrededor del mundo y, particularmente, en Latinoamérica (Nordgren, 2011; Pérez et al. 2010; Puenayán, 2009); el reconocimiento de los saberes, las creencias y las prácticas que tienen las comunidades con respecto a estos factores, contribuyen a llenar vacíos en la información científica y preparan el camino para el diseño de medidas de mitigación y adaptación al cambio climático (Pinilla et al. 2012; Correa et al. 2012; Ulloa, 2011).

Para Urbina \& Martínez (2006), la percepción es el primer proceso cognoscitivo del ser humano, es la visión personal del mundo mezclada con la fantasía que cada sujeto posee y el carácter egocéntrico de la experiencia y de la visión personal, así como la influencia de la estructura social, el contexto cultural y el lenguaje en la formación de determinadas pautas básicas colectivas. Capel (1973) plantea que la percepción humana tiene un papel decisivo en la formación de una imagen del medio real, la cual, influye directamente sobre su comportamiento y, por lo tanto, en su relación con la naturaleza.

Uno de los aspectos más importantes para entender las manifestaciones del cambio climático en una comunidad es adentrarse en el conocimiento de las opiniones que tienen sus habitantes sobre el problema y así, comprender sus intereses, demandas y necesidades. Lo anterior, facilitará el establecimiento de las bases de construcción de los procesos de participación social (Soares \& Gutiérrez, 2011; Nordgren, 2011; Echeverri, 2009). Al respecto, la sicología ambiental ha realizado aportes significativos al tema de la percepción humana sobre el medio natural y el cambio climático, al comportamiento ambiental relacionado con las variables socio-demográficas, como nivel de ingreso y la edad y acerca de la reacción social al cambio climático (Gifford et al. 2011; Uzzell, 2008; Stern, 2000). De esta manera, en las comunidades campesinas e indígenas, los efectos del cambio climático, probablemente, son más evidentes, a partir de la percepción, debido a su interrelación cotidiana con la naturaleza y sus recursos (Ramos et al. 2011).

La atención mundial sobre el cambio climático ha estado orientada a aspectos relacionados con las causas naturales o antrópicas, enfocándose en quiénes tienen las responsabilidades políticas y económicas, a nivel de país y sectores privados (Echeverri, 2009; Conde \& Saldaña, 2007; Foster, 2001). Por ejemplo, O'connor et al. (1999), investigaron para la Agencia de Protección Ambiental de Estados Unidos, la relación entre la percepción de riesgo y la voluntad comunitaria y política de abordar las problemáticas asociadas con el cambio climático. En contraste, los aspectos sociales, a nivel local, han tenido menos relevancia (Soares \& Gutiérrez, 2011). Para Nordgren (2011) y Ulloa (2011) es importante conocer la percepción de las comunidades locales del cambio climático y tener en cuenta el diseño de estrategias con los actores clave, pues a partir del conocimiento local es posible darles viabilidad a sus estrategias de adaptación.

Conde \& Lonsdale (2005) señalan que uno de los retos más conocidos para la investigación del cambio climático es el de bajar la escala espacial, con el fin de tener escenarios de evaluación regionales y locales. El reconocimiento de los saberes, las creencias y las prácticas que tienen las comunidades con respecto a estos factores, contribuyen no solamente a llenar vacíos en la información científica, sino que preparan el camino para el diseño de medidas de adaptación. Así que, se hace necesaria una revisión de las investigaciones sobre las percepciones del cambio climático, a nivel latinoamericano.

Desde la perspectiva de las ciencias sociales, el clima es el resultado de la forma en que los individuos perciben, se apropian e interpretan los eventos meteorológicos y climáticos que ocurren a su alrededor. Según Mariño (2011), el concepto de clima es una construcción cultural que se elabora a partir de procesos materiales y simbólicos y que denota aspectos culturales, espaciales e históricos.

Los enfoques metodológicos utilizados en los estudios de percepción de cambio climático son el cuantitativo y el cualitativo, en donde los dos métodos se complementan (Retamal et al. 2011). Los principales instrumentos utilizados para recolectar información son las encuestas, as entrevistas semiestructuradas y la observación directa, los cuales, se 
contrastan y analizan con fuentes de información secundarias (De los Ríos \& Almeida, 2011; Nordgren, 2011; Soares \& Gutiérrez, 2011; Echeverri, 2009). Para Hurtado (2000) y Hernández (1997), esta combinación de métodos cualitativos, cuantitativos, técnicas y propósitos es valiosa, porque más allá de la discusión epistemológica, la construcción del conocimiento está siendo abordada de manera integral, holística y sistémica entre ciencias duras y blandas.

En general, la percepción de las diferentes comunidades locales es que existe un incremento de la temperatura y una disminución de la precipitación en las diferentes zonas de estudio (Sierra, 2011; Ulloa, 2011; Echeverri, 2009; Puenayán, 2009). Al respecto, numerosos estudios de percepción de cambio climático se han realizado en comunidades indígenas de diversos países: Nordgren (2011), en Bolivia; Echeverri (2009), en el Amazonas Colombiano y Puenayán (2009), en Nariño. Igualmente, se destacan las investigaciones financiadas por organismos internacionales, como El Banco Mundial. Así, se ha reportado un incremento en las tormentas intensas y huracanes en Meso-américa y el Caribe, cambios en la precipitación y la estacionalidad en el Amazonas colombiano y una escasez de agua para usos agropecuarios, erosión de ecosistemas y cambios en la biodiversidad en la región de Los Andes (Kronik \& Verner, 2010a).

Adicionalmente, se han realizado estudios de las estrategias desplegadas por los pueblos indígenas y las comunidades afro-descendientes, para afrontar el cambio climático (Tocancipá, 2013; Ulloa, 2011; Dounias, 2011). Al respecto, Kronik \& Verner (2010b) reportaron que las comunidades indígenas latinoamericanas, frecuentemente, no lograban encontrar una respuesta a sus necesidades a partir de su conocimiento y estrategias de adaptación cultural; una problemática que se intensificó por la falta de apoyo institucional del Estado. Por lo tanto, las percepciones como las formas de adaptación están determinadas por factores ligados a la cultura de cada sociedad e, igualmente, por factores de tipo ambiental e institucional (Dounias, 2011; Ulloa et al. 2008). De esta manera, la elección de las estrategias está condicionada por el contexto en que viven los individuos, es parte de un tejido cultural y se relaciona con otras estrategias locales de subsistencia (De los Ríos y Almeida, 2011; Martín, 2010; Bonatti, 2007).

El objetivo principal de la investigación fue realizar una revisión de los estudios de percepción de cambio climático que se han desarrollado en Latinoamérica, durante los últimos quince años (1997-2012), en comunidades locales campesinas e indígenas. Adicionalmente, se pretende indagar acerca de qué metodologías y herramientas de trabajo han sido utilizadas para evaluar la percepción de las comunidades locales con respecto al cambio climático, para finalmente, conocer cuáles han sido sus estrategias de adaptación.

\section{MATERIALES Y MÉTODOS}

Sistema de búsqueda bibliográfica: Se utilizó una metodología de búsqueda sistemática de los estudios realizados en Latinoamérica, durante los últimos quince años, acerca de la percepción del cambio climático en comunidades locales campesinas e indígenas. Las bases de datos utilizadas para detectar los documentos, tales como artículos y libros, fueron Google Académico, la Red de Revistas Científicas de América Latina y el Caribe, España y Portugal (Redalyc) y el Catálogo de Búsqueda de la Biblioteca Pública Luis Ángel Arango (BLAA) (Bogotá D.C., Colombia). Adicionalmente, se utilizaron las siguientes combinaciones de palabras clave en español: "percepción", "variabilidad climática" y "comunidades locales"; "percepción», "cambio del clima» y «comunidades locales» y "estrategias de adaptación", "cambio climático" y "comunidades locales". Los documentos detectados se limitaron, espacialmente y temporalmente, a Latinoamérica, entre 1997-2012.

Posteriormente, se seleccionaron los documentos que hubiesen definido explícitamente la percepción de las comunidades locales con respecto al cambio climático en Latinoamérica. En este sentido, se establecieron durante el análisis bibliográfico los siguientes criterios de exclusión, para los documentos detectados: (i) los que sustentaron el cambio climático desde un punto de vista técnico; (ii) los relacionados con el análisis estadístico de la variabilidad climática; (iii) los de evaluación del impacto de los eventos extremos asociados al cambio climático; (iv) los de políticas sobre el cambio climático y, (v) los que documentaron el cambio climático como un fenómeno global.

Análisis bibliográfico: Se cuantificaron por base de datos los 1.489 documentos hallados. En este sentido, la base de datos que detectó el mayor número de documentos fue Redalyc (60,7\%), seguida de Google Académico $(37,2 \%)$ y BLAA (2,01\%). Por otro lado, la combinación de palabras clave con un mayor número de coincidencias fue "percepción», «cambio del clima» y "comunidades locales» (46,5\%); seguida de "percepción", "variabilidad climática" y "comunidades locales" (32,5\%) y de la combinación de palabras clave "estrategias de adaptación", "cambio climático" y "comunidades locales" (21\%). Lo anterior, en promedio para todas las bases de datos utilizadas.

Posteriormente, se realizó una revisión de los resúmenes o índices de todos los documentos detectados, para evaluar su pertinencia en la presente investigación bibliográfica; lo anterior, a partir de los criterios de exclusión establecidos en el apartado anterior. De esta manera, se descartó un 94,7\%, $94,2 \%$ y $86,1 \%$ de los documentos detectados por las bases de datos Redalyc, Google Académico y BLAA, respectivamente. Por lo tanto, en la construcción del presente artículo 
de revisión bibliográfica, se seleccionó un total de 62 documentos, entre artículos y libros; representando un 4,2\% de los documentos detectados inicialmente por las bases de datos utilizadas.

Finalmente, se desarrolló una última fase de análisis con la bibliografía detectada. Se estudiaron y clasificaron las temáticas asociadas y reportadas por los resúmenes e índices de los documentos, con el objeto de establecer categorías y sub-categorías de análisis. Por lo tanto, se identificaron tres categorías principales de estudio: (i) metodologías y herramientas de trabajo $(49,1 \%)$; (ii) percepciones de cambio climático $(64,2 \%)$ y, (iii) estrategias de adaptación $(98,1 \%)$. Por último, el análisis bibliográfico permitió detectar 13 subcategorías de estudio (Tabla 1).

\section{RESULTADOS Y DISCUSIÓN}

Metodologías y herramientas de trabajo: La investigación bibliográfica evidenció la existencia de tres enfoques metodológicos en los estudios de percepción del cambio climático en comunidades locales: (i) cuantitativo, (ii) cualitativo y, (iii) mixto. El enfoque cuantitativo aportó datos matemáticamente medibles. Por su parte, el enfoque cualitativo se aproximó al problema desde una perspectiva de los valores ambientales, sociales, económicos y culturales de cada comunidad local campesina e indígena.

En relación con lo anterior, Gay (2006) planteó que el reconocimiento individual sobre el cambio del clima fue básicamente cualitativo, por lo que los cambios de comportamiento de

Tabla 1. Categorías de análisis para la información bibliográfica seleccionada.

\begin{tabular}{|c|c|c|c|}
\hline Categoría & \multicolumn{2}{|c|}{$\begin{array}{l}\text { Documentos (\%) } \\
\quad(n=53)\end{array}$} & Sub-categoría \\
\hline \multirow{3}{*}{$\begin{array}{l}\text { Metodologías y } \\
\text { herramientas de } \\
\text { trabajo }\end{array}$} & \multirow{3}{*}{$\begin{array}{c}49,1 \\
(n=26)\end{array}$} & 92,3 & (i) Metodologías cualitativas \\
\hline & & 7,7 & (ii) Metodologías cuantitativas \\
\hline & & 100 & (iii) Herramientas de recolección de información \\
\hline \multirow{7}{*}{$\begin{array}{l}\text { Percepción de } \\
\text { cambio climático }\end{array}$} & \multirow{7}{*}{$\begin{array}{c}64,2 \\
(n=34)\end{array}$} & 85,3 & (i) Precipitación (Largo plazo) \\
\hline & & 91,2 & (ii) Temperatura (Largo plazo) \\
\hline & & 94,1 & (iii) Variabilidad climática (Corto plazo) \\
\hline & & 44,1 & (iv) Recurso hídrico \\
\hline & & 8,8 & (v) Paisaje \\
\hline & & 55,9 & $\begin{array}{l}\text { (vi) Hábitos culturales (p.ej., alimentación, vestimenta y } \\
\text { actividades económicas, sociales y culturales) }\end{array}$ \\
\hline & & 20,6 & $\begin{array}{l}\text { (vii) Otras percepciones (p.ej., descenso de glaciares, } \\
\text { pérdida de humedad en el suelo, aparición de nuevas } \\
\text { plagas y enfermedades) }\end{array}$ \\
\hline \multirow{3}{*}{$\begin{array}{l}\text { Estrategias de } \\
\text { adaptación }\end{array}$} & \multirow{3}{*}{$\begin{array}{c}98,1 \\
(n=52)\end{array}$} & 57,7 & (i) Agropecuaria \\
\hline & & 40,4 & (ii) Recurso hídrico \\
\hline & & 48,1 & $\begin{array}{l}\text { (iii) Comunitarias (p.ej., capacitación, cooperación, } \\
\text { asociaciones campesinas y conocimiento ancestral) }\end{array}$ \\
\hline
\end{tabular}

los actores locales solamente se pudieron visualizar mediante la valoración subjetiva que ellos tienen con respecto al tema, como por ejemplo, el estilo de vida, las concepciones del bienestar y las consecuencias de los efectos del cambio climático. Por esto, la revisión mostró que la información obtenida para evaluar la percepción que tienen las comunidades locales acerca del cambio climático se ha adquirido mediante la combinación de métodos cualitativos y cuantitativos (Pinilla et al. 2012; Dounias, 2011; Nordgren, 2011;
Sierra, 2011; Tupaz \& Guzmán, 2011; Echeverri, 2009); sin embargo, al tratarse de estudios de percepción del cambio climático, las investigaciones tuvieron preferencia por el enfoque cualitativo.

En este sentido, Hernández (1997) reportó que el enfoque cualitativo reconoció la naturaleza holística de la construcción del conocimiento, porque a través de la integración de las concepciones e interpretaciones de los actores se pu- 
dieron formular las estrategias de adaptación. Por lo tanto, esta gama de técnicas cualitativas son particularmente útiles cuando el fenómeno a estudiar es difícil de medir, como ocurre con la percepción del cambio climático en comunidades locales, tales como las campesinas e indígenas.

Adicionalmente, se reportó que la investigación cualitativa de la percepción hizo referencia a las personas como eje central de la dinámica eco-social del territorio (Nordgren, 2011; Echeverri, 2009; Bonatti, 2007). A partir de lo anterior, se sugirió que las técnicas de investigación cualitativas resultaron de gran utilidad para investigar cómo el cambio del clima fue comprendido, experimentado, interpretado por las personas y cómo sus conductas, interacciones, perspectivas y prácticas repercutieron en la formulación o utilización de estrategias de adaptación (Pinilla et al. 2012; Soares \& Gutiérrez, 2011; Oltra et al. 2009; Magaña, 2004).

La investigación bibliográfica mostró que el 92,3\% de los documentos seleccionados utilizaron, principalmente, la estrategia metodológica cualitativa; no obstante, también se observó en estos documentos, pequeños aportes de la estrategia cuantitativa. En este sentido, Hurtado (2000) y Hernández (1997) reportaron que esta combinación de métodos era útil, pues la construcción del conocimiento fue abordada de manera integral, holística y sistémica, entre las ciencias duras y blandas y, por otro lado, este nuevo enfoque tuvo sus raíces en la investigación cualitativa, en la cual, se reconoció la subjetividad del sujeto investigado, la propia subjetividad del investigador y en donde la construcción del conocimiento fue compartida por ellos.

Por otro lado, la revisión bibliográfica permitió evidenciar que una de las herramientas de trabajo más utilizadas para la recolección de los datos cualitativos fue la entrevista semiestructurada (Tabla 2). En este sentido, el 76,9\% de los documentos seleccionados utilizó esta herramienta de trabajo. El objetivo de su aplicación radicó en obtener información cualitativa, para profundizar en el análisis de la percepción del cambio climático y en su explicación, por parte de las comunidades locales (Sierra, 2011; Ramos et al. 2011; De los Ríos \& Almeida, 2011; Echeverri, 2009; Lozano, 2004).

Adicionalmente, la investigación evidenció que la encuesta fue otra herramienta de trabajo utilizada en los estudios de percepción de cambio climático en comunidades locales; en este sentido, el $42,3 \%$ de los documentos seleccionados utilizó esta herramienta. Los estudios que utilizaron exclusivamente encuestas, mostraron que su aplicación fue importante para realizar un barrido general de las percepciones de las comunidades locales con respecto al clima y sus variaciones (Pinilla et al. 2012; Soares \& Gutiérrez, 2011; Vignola, 2010); sin embargo, para otros investigadores, esta herramienta presentó limitaciones, ya que se pudo incurrir en un sesgo por su aplicación estática, es decir, limitó las respuestas que pudieron dar las personas encuestadas (Nordgren, 2011; Tocancipá et al. 2011; Echeverri, 2009).

Las siguientes metodologías participativas fueron otras herramientas detectadas en la investigación bibliográfica: talleres comunitarios, grupos focales, mingas, narraciones y testimonios (Correa et al. 2012; Martín, 2010; Pérez et al. 2010; Yana, 2008; García et al. 2008; Gay et al. 2007). Éstas, consistieron en visitas a las comunidades locales de estudio para aplicar las herramientas mencionadas y aplicar una nueva herramienta, la observación en campo; lo anterior, con el objeto de extraer la información cualitativa asociada a la temática de investigación (Sierra; 2011; De los Ríos \& Almeida, 2011; Puenayán, 2009). A partir de lo anterior, la investigación bibliográfica mostró que $30,1 \%$ y $30,8 \%$ de los documentos seleccionados, utilizaron como herramientas de trabajo, las metodologías participativas y la observación en campo, respectivamente.

Asimismo, se detectó que el conocimiento local o propio del investigador y el enfoque de género fueron herramientas complementarias en la recolección de los datos cualitativos sobre la percepción del cambio climático en comunidades locales (Nordgren, 2011; García et al. 2011; Echeverri, 2009; Puenayán, 2009); lo anterior, con el objeto de evidenciar los aspectos de la vida cotidiana y poder compararlos con la información obtenida por la utilización de las herramientas mencionadas inicialmente, es decir, la entrevista semi-estructurada y la encuesta.

La investigación mostró, en orden de importancia, que el $76,9 \%, 42,3 \%, 30,8 \%, 30,1 \%, 23,1 \%$ y $15,4 \%$ de los documentos seleccionados, utilizó como herramientas de trabajo la entrevista semi-estructurada, la encuesta, la observación en campo, las metodologías participativas, el conocimiento local y el enfoque de género, respectivamente (Tabla 2). Conde \& Lonsdale (2005) reportaron que la investigación social complementada con las anteriores herramientas permitió el reconocimiento de diferentes realidades y prácticas sociales asociadas a la percepción del cambio climático, en comunidades locales. Además, los resultados mostraron que los estudios latinoamericanos de percepción del cambio climático, con el uso de las herramientas en mención, fueron realizados, principalmente, en comunidades locales campesinas $(65,4 \%)$, antes que en comunidades indígenas (34,6\%) (Tabla 2).

Finalmente, Gay et al. (2006) plantearon que siendo estudios que se desarrollaron a partir de una muestra de percepciones es importante interpretar los resultados con precaución y de manera crítica y es, por este motivo, que se necesita contrastar y analizar las percepciones y los testimonios obtenidos con otras fuentes de información secundaria. 
Tabla 2. Herramientas utilizadas en los estudios de percepción de cambio climático en comunidades locales.

\begin{tabular}{|c|c|c|c|c|c|c|c|c|c|}
\hline \multirow{2}{*}{$\begin{array}{l}\text { Investigaciones } \\
\qquad(n=26)\end{array}$} & \multirow{2}{*}{ Localización } & \multicolumn{2}{|c|}{ Comunidad $^{\mathrm{a}}$} & \multicolumn{6}{|c|}{ Herramienta } \\
\hline & & C & I & Es & Oc & $\mathrm{Cl}$ & $\mathrm{E}$ & Mp & $\mathrm{Eg}$ \\
\hline Bonatti (2007) & Anchieta y Santa Catarina (Brasil) & $\mathrm{x}$ & & $\mathrm{x}$ & $\mathrm{x}$ & & & & \\
\hline Correa et al. (2012) & $\begin{array}{l}\text { Sapzurro y Acandí. Urabá chocoano } \\
\text { (Colombia) }\end{array}$ & $\mathrm{x}$ & & $\mathrm{x}$ & & & & & \\
\hline $\begin{array}{l}\text { De los Ríos \& Almeida } \\
\text { (2011) }\end{array}$ & $\begin{array}{l}\text { Páramo de Sonsón. Antioquia } \\
\text { (Colombia) }\end{array}$ & $\mathrm{x}$ & & $\mathrm{x}$ & $\mathrm{x}$ & & & & \\
\hline Echeverri (2009) & $\begin{array}{c}\text { Amazonas, Caquetá y Putumayo } \\
\text { (Colombia) }\end{array}$ & & $\mathrm{x}$ & $\mathrm{x}$ & & $\mathrm{X}$ & & & \\
\hline García et al. (2011) & Toribío. Cauca (Colombia) & & $\mathrm{x}$ & $\mathrm{x}$ & & $\mathrm{x}$ & & & $\mathrm{x}$ \\
\hline Gay et al. (2007) & Hermosillo. Sonora (México) & $\mathrm{x}$ & & $\mathrm{x}$ & & & $\mathrm{x}$ & $\mathrm{x}$ & \\
\hline Gay (2006) & Jalisco, Colima y Michoacán (México) & $\mathrm{x}$ & & $\mathrm{x}$ & & & $\mathrm{x}$ & $\mathrm{x}$ & \\
\hline Gay et al. (2006) & Veracruz (México) & $\mathrm{x}$ & & $\mathrm{x}$ & $\mathrm{x}$ & & & $\mathrm{x}$ & \\
\hline Martín (2010) & Puracé. Cauca (Colombia) & & $\mathrm{x}$ & & & $\mathrm{x}$ & & $\mathrm{x}$ & \\
\hline Nordgren (2011) & $\begin{array}{c}\text { La Paz, Santa Cruz, Chuquisaca, Beni, } \\
\text { Pando y Cochabamba (Bolivia) }\end{array}$ & $\mathrm{x}$ & $\mathrm{x}$ & $\mathrm{x}$ & $\mathrm{x}$ & $\mathrm{x}$ & & $\mathrm{x}$ & \\
\hline $\begin{array}{l}\text { Novión \& Estrada } \\
\text { (2011) }\end{array}$ & Punta Arenas. Magallania (Chile) & $\mathrm{x}$ & & & & & $\mathrm{x}$ & & \\
\hline Pérez (2007) & Guatemala (Guatemala) & & $\mathrm{x}$ & $\mathrm{x}$ & & $\mathrm{x}$ & & & $\mathrm{x}$ \\
\hline Pérez et al. (2010) & Andes de Bolivia, Ecuador y Perú & $\mathrm{x}$ & & & & & $\mathrm{x}$ & & \\
\hline Pinilla et al. (2012) & $\begin{array}{c}\text { Betulia, San Vicente, Zapatoca, Girón y } \\
\text { Lebrija. Santander (Colombia) }\end{array}$ & $\mathrm{x}$ & & & & & $\mathrm{x}$ & & \\
\hline Puenayán (2009) & Resguardo Panán. Nariño (Colombia) & & $\mathrm{x}$ & $\mathrm{x}$ & $\mathrm{x}$ & & & $\mathrm{x}$ & \\
\hline Ramos et al. (2011) & Comunidad Nasa. Cauca. (Colombia) & & $\mathrm{x}$ & $\mathrm{x}$ & & & & $\mathrm{x}$ & \\
\hline Sierra (2011) & $\begin{array}{c}\text { Sierras Nevadas de Santa Marta y del } \\
\text { Cocuy, y Cauca. (Colombia) }\end{array}$ & & $\mathrm{x}$ & $\mathrm{x}$ & $\mathrm{x}$ & & & & \\
\hline $\begin{array}{l}\text { Soares \& Gutiérrez } \\
(2011)\end{array}$ & San Felipe. Yucatán (México) & $\mathrm{x}$ & & $\mathrm{x}$ & $\mathrm{x}$ & & $\mathrm{x}$ & & \\
\hline Tocancipá et al. (2011) & Guapi. Cauca (Colombia) & $\mathrm{x}$ & & $\mathrm{x}$ & & & & & \\
\hline Treulen (2008) & Región de la Araucanía (Chile) & & $\mathrm{x}$ & $\mathrm{x}$ & & $\mathrm{x}$ & & & $\mathrm{x}$ \\
\hline Tupaz \& Guzmán (2011) & Nudo de los Pastos. Nariño (Colombia) & & $\mathrm{x}$ & & $\mathrm{x}$ & & $\mathrm{x}$ & & \\
\hline Ulloa \& Prieto (2012) & $\begin{array}{c}\text { Bosa y Usaquén. Bogotá D.C. (Colom- } \\
\text { bia) }\end{array}$ & $\mathrm{x}$ & & $\mathrm{x}$ & & & $\mathrm{x}$ & & \\
\hline Van Der Mollen (2011) & Cotacachi. (Ecuador) & $\mathrm{x}$ & & $\mathrm{x}$ & & & $\mathrm{x}$ & & \\
\hline Vignola (2010) & $\begin{array}{c}\text { Área Metropolitana. San José de Costa } \\
\text { Rica (Costa Rica) }\end{array}$ & $\mathrm{x}$ & & & & & $\mathrm{x}$ & & \\
\hline Viella (1997) & $\begin{array}{c}\text { Distritos: Álvarez, La Montaña, Morelos } \\
\text { y Zaragoza, México (México) }\end{array}$ & $\mathrm{x}$ & & $\mathrm{x}$ & & & $\mathrm{x}$ & & \\
\hline Yana (2008) & Umala y Ancoraimes. La Paz (Bolivia) & $\mathrm{x}$ & & $\mathrm{x}$ & & & & $\mathrm{X}$ & $\mathrm{x}$ \\
\hline & Proporción (\%) & 65,4 & 34,6 & 76,9 & 30,8 & 23,1 & 42,3 & 30,1 & 15,4 \\
\hline
\end{tabular}

Nota. ${ }^{\mathrm{a}} \mathrm{C}$ : campesina; I: indígena. ${ }^{\text {}}$ Es: entrevista semi-estructurada; Oc: observación en campo; Cl: conocimiento local; E: encuesta; Mp: metodologías participativas (Grupos focales, talleres, mingas, testimonios y narraciones locales); Eg: enfoque de género. 
Lo anterior, con el fin de analizar con mayores elementos su representatividad regional y posibilitar una mejor base de discusión para el establecimiento de conclusiones, como lo hicieron Pinilla et al. (2012), Sierra (2011), Nordgren (2011), De los Ríos \& Almeida (2011), y Echeverri (2009).

Percepciones Latinoamericanas de cambio climático: Durante las últimas décadas en América Latina, se han reportado cambios en la precipitación y en la temperatura (López \& Cadena, 2010; Costa, 2007; Gay et al. 2006; Conde \& Lonsdale, 2005; Pabón, 2003). La investigación bibliográfica mostró que la percepción habitual en las comunidades locales latinoamericanas fue que existió un incremento de la temperatura (Soares \& Gutiérrez, 2011; García et al. 2011; Dounias, 2011; Pérez, 2007; Viella, 1997); sin embargo, en Bolivia, algunas comunidades locales percibieron un descenso de la temperatura (Nordgren, 2011). A partir de lo anterior, Nordgren (2011) reportó que esta diferencia en la percepción del clima sugirió la existencia de fenómenos regionales de variabilidad climática en los valles de Cochabamba (Bolivia).

Adicionalmente, Yana (2008) reportó que la percepción de cambio climático en las mujeres indígenas bolivianas estuvo principalmente asociada a una reducción de la humedad del suelo. Asimismo, Pérez (2007) reportó que las comunidades indígenas de Guatemala manifestaron que el impacto del cambio climático se evidenció en una reducción de la humedad de los suelos y, por lo tanto, en una reducción del agua disponible para los cultivos, el ganado y la vegetación nativa de las comunidades locales en estudio. En este sentido, Pérez et al. (2010) describieron cómo el cambio climático afectó el sector agropecuario de algunas comunidades locales de Bolivia, Ecuador y Perú; lo anterior, a través del retroceso de los glaciares, de cambios en los patrones hidrológicos y por la aparición de nuevas plagas y enfermedades en los cultivos y el ganado. Por lo tanto, algunas de las comunidades locales latinoamericanas han percibido una alteración de los ciclos climáticos en su intensidad y distribución temporal y espacial (Correa et al. 2012; García et al. 2011; De los Ríos \& Almeida, 2011; Echeverri, 2009; Treulen, 2008).

Las anteriores percepciones y evidencias en las comunidades locales fueron en contra de su forma de vida tradicional, por lo que se generó un desequilibrio en la organización familiar y social, en especial, en las comunidades indígenas de la Amazonia y el Macizo Colombiano, así como en las comunidades campesinas andinas (Sierra, 2011; Pérez et al. 2010; Puenayán, 2009; Echeverri, 2009; Pérez, 2007).

Los desequilibrios a nivel familiar ocurrieron por los elevados niveles de pobreza, lo que obligó a las comunidades locales a emigrar hacia la ciudad, en busca de mejores condiciones económicas (De los Ríos \& Almeida, 2011; Treulen, 2008). De esta manera, a nivel social, cambió el rol de cada persona en la comunidad local, principalmente, en las actividades asociadas con la agricultura, pesca y caza (García et al. 2011; Pérez et al. 2010; Echeverri, 2009). Por último, Echeverri (2009) y Donato (2007) reportaron que las percepciones e impactos negativos asociados al cambio climático que se combinaron con la explotación de los recursos naturales y el conflicto armado, generaron mayor vulnerabilidad en las comunidades locales.

La tabla 1 presenta las percepciones identificadas en las comunidades locales con respecto al cambio climático, a partir de los documentos detectados y de las sub-categorías de análisis, establecidas en la investigación bibliográfica. Como se puede observar, la percepción de variabilidad climática fue la que asoció el mayor número de documentos $(94,1 \%)$; seguida en orden de magnitud por: incremento de la temperatura $(91,2 \%)$, cambio en la precipitación (85,3\%), cambio en los hábitos culturales $(55,9 \%)$, escasez del recurso hídrico $(44,1 \%)$ y otras percepciones: descenso de glaciares, pérdida de humedad en el suelo, aparición de nuevas plagas y enfermedades $(20,6 \%$ y cambios en el paisaje $(8,8 \%)$. Por lo tanto, los resultados sugirieron que la percepción dominante en las comunidades locales campesinas e indígenas fue que existió un cambio asociado, principalmente, con el factor climático; en este sentido, el factor climático representó en promedio un 90,2\% de los documentos detectados, durante la investigación bibliográfica (Tabla 1).

Por otro lado, Ulloa (2011) reportó que si bien la población rural identificó las alteraciones ocurridas en el comportamiento normal del clima en los últimos años, el cambio climático continuó siendo un tema desconocido en las comunidades campesinas e indígenas; sin embargo, Fernández \& Mancinas (2012), y Urbina \& Martínez (2006) evidenciaron la existencia de un acceso a la información, a través de emisoras locales, periódicos y la televisión, que influyeron, de manera significativa, en el conocimiento que se tuvo sobre el cambio climático en las comunidades locales.

Finalmente, debido a la complejidad del tema y a la escala temporal en la que ocurrió el fenómeno de cambio climático, la población campesina tuvo una imagen más estática de la situación; por el contrario, en las comunidades indígenas, se tuvo una visión más dinámica de la naturaleza (García et al. 2011; Echeverri, 2009; Pérez, 2007; Viella, 1997). Al respecto, Ulloa (2011) mencionó que el conocimiento de la dinámica natural pudo llevar a tendencias que generaron escenarios dramáticos o pesimistas, que desincentivaron las actitudes proactivas y preventivas con respecto a los procesos y las estrategias de adaptación al cambio climático. 
Estrategias de adaptación: Las percepciones detectadas y las estrategias de adaptación al cambio climático estuvieron determinadas por factores ligados a la cultura de cada comunidad local, igualmente, a factores de tipo ambiental e institucional (Ulloa et al. 2008; Magrin et al. 2007). En este sentido, las estrategias de adaptación fueron actos conscientes y racionales de los individuos que estuvieron contenidas dentro del límite de cada estructura social (De los Ríos \& Almeida, 2011; Martín, 2010; Bonatti, 2007). Por lo tanto, la elección de las estrategias adaptación estuvo condicionada por el contexto en el que vivían los individuos y fue parte de un tejido cultural que se relacionó con otras estrategias locales de subsistencia (De los Ríos \& Almeida, 2011; Martín, 2010; Bonatti, 2007; PNUD, 2007).

Adicionalmente, se evidenció, en la presente investigación, que los datos recolectados con las herramientas de trabajo detectadas, tales como la entrevista, los talleres y los grupos focales, fueron contrastados y complementados con datos e información secundaria, obtenida de revisiones bibliográficas, por ejemplo, de variables climatológicas y estadísticas (De los Ríos \& Almeida, 2011; Pérez et al. 2010; Gay et al. 2007; Puenayán, 2009; Yana, 2008). Lo anterior, para la formulación de estrategias de adaptación al cambio climático, por parte de las comunidades campesinas e indígenas.

Los resultados indican que las estrategias de adaptación al cambio climático en comunidades locales se agrupan en tres categorías principales: (i) agropecuaria, (ii) hídrica y (iii) comunitaria (Tabla 3). El 57,6\% de los documentos detectados correspondieron a estrategias de adaptación asociadas con la categoría agropecuaria. En este sentido, los investigadores del Programa de las Naciones Unidas para el Desarrollo (PNUD) reportaron que las estrategias de adaptación asociadas con el sector agropecuario fueron claves para consolidar la seguridad alimentaria de las comunidades rurales de Los Andes colombianos y latinoamericanos (PNUD, 2007).

Por otro lado, los resultados mostraron que, en segundo lugar, se ubicaron las estrategias de adaptación asociadas con la categoría comunitaria, representando un $48,1 \%$ de los documentos detectados (Tabla 3). Los reportes investigativos estuvieron principalmente asociados con resguardos o comunidades indígenas, donde existió un mayor conocimiento y aplicación de los saberes tradicionales (Sierra, 2011; Puenayán, 2009; Echeverri, 2009).

En tercer lugar, se ubicaron las estrategias de adaptación asociadas con la categoría hídrica, representando un 40,4\%, de los documentos detectados. En este sentido, Magrin et al. (2007) y Conde \& Lonsdale (2005) señalaron que Latinoamérica atraviesa por un conjunto de problemas asociados con el recurso hídrico, debido a su distribución temporal y espacial no uniforme, dificultando el manejo y uso por parte de las comunidades campesinas e indígenas. Por lo tanto, la investigación bibliográfica evidenció que las estrategias de adaptación se enfocaron en la implementación de acciones de orden técnico, para optimizar el aprovechamiento del recurso hídrico (Tabla 3).

Por último, Pérez et al. (2010) reportaron que no existió una concepción universal y única para el proceso de adaptación al cambio climático, ya que la situación individual de cada comunidad local demandó el desarrollo de estrategias particulares. En el desarrollo de las estrategias de adaptación jugaron un papel decisivo los efectos observados y esperados del cambio, así como los recursos naturales, el entorno institucional y la estructura económica y poblacional de las comunidades campesinas e indígenas.

Conclusiones: La investigación bibliográfica muestra la existencia de tres enfoques metodológicos en los estudios de percepción del cambio climático en comunidades locales latinoamericanas: (i) cuantitativo, (ii) cualitativo y (iii) mixto. En este sentido, el 92,3\% de los documentos seleccionados utilizan, principalmente, la estrategia metodológica cualitativa, con pequeños aportes de la estrategia cuantitativa. Además, se evidencia en orden de magnitud que las principales herramientas utilizadas en las investigaciones de percepción del cambio climático en comunidades locales latinoamericanas son: (i) entrevista semi-estructurada (76,9\%); (ii) encuesta (42,3\%); (iii) observación en campo (30,8\%); (iv) metodologías participativas $(30,1 \%)$; (v) conocimiento local $(23,1 \%)$ y (vi) enfoque de género $(15,4 \%)$. Los resultados muestran que los estudios latinoamericanos de percepción del cambio climático con el uso de las herramientas en mención fueron realizados, principalmente, en comunidades locales campesinas $(65,4 \%)$, antes que en comunidades indígenas $(34,6 \%)$.

Por otro lado, las percepciones de cambio climático en comunidades locales identificadas durante la investigación bibliográfica, en orden de magnitud, son las siguientes: (i) variabilidad climática $(94,1 \%)$; (ii) incremento de temperatura (91,2\%); (iii) cambio en la precipitación (85,3\%); (iv) cambio en los hábitos culturales (55,9\%); (v) escasez del recurso hídrico $(44,1 \%)$ y (vi) otras percepciones: descenso de glaciares, pérdida de humedad en el suelo, aparición de nuevas plagas y enfermedades $(20,6 \%)$ y cambios en el paisaje $(8,8 \%)$. De esta manera, los resultados sugirieren que la percepción dominante en las comunidades locales campesinas e indígenas latinoamericanas es que existe un cambio asociado, principalmente, con el factor climático; lo anterior, representado por un 90,2\% de los documentos detectados durante la investigación bibliográfica.

Los resultados obtenidos muestran que las estrategias de adaptación al cambio climático en comunidades locales latinoamericanas se pueden agrupar en tres categorías: (i) 


\begin{tabular}{|c|c|c|c|c|c|c|c|c|c|c|c|c|c|c|c|c|}
\hline \multirow{2}{*}{ 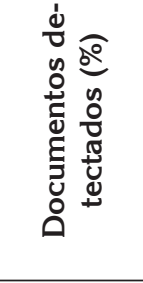 } & $\begin{array}{l}\text { : } \\
\text { in }\end{array}$ & $\begin{array}{l}0 \\
\stackrel{+}{+}\end{array}$ & $\begin{array}{l}0 \\
\stackrel{0}{\circ}\end{array}$ & $\stackrel{m}{m}$ & $\stackrel{m}{m}$ & $\hat{\tilde{\omega}}$ & $\begin{array}{l}m \\
m\end{array}$ & $\stackrel{m}{\vartheta}^{m}$ & $\begin{array}{l}\text { ̊े } \\
\text { f }\end{array}$ & $\begin{array}{l}\text { ̊े } \\
\text { f }\end{array}$ & $\begin{array}{c}m \\
\underset{J}{-}\end{array}$ & $\vec{\sigma}$ & $\begin{array}{l}0 \\
\dot{f}\end{array}$ & $\begin{array}{l}0 \\
\dot{m}\end{array}$ & $\begin{array}{l}\circ \\
\infty \\
\infty\end{array}$ & $\begin{array}{l}0 \\
\dot{q}\end{array}$ \\
\hline & \multicolumn{8}{|c|}{$\begin{array}{ll}o & \widehat{m} \\
\hat{n} & \\
\text { in } & \| \\
& \Xi\end{array}$} & \multicolumn{4}{|c|}{$\begin{array}{ll}\nabla & \vec{N} \\
\dot{\sigma} & \| \\
& \Xi\end{array}$} & \multicolumn{4}{|c|}{ 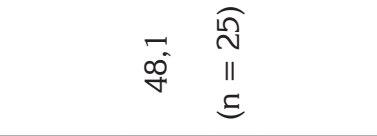 } \\
\hline 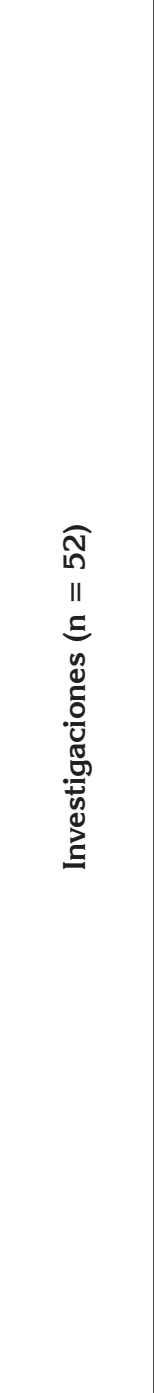 & 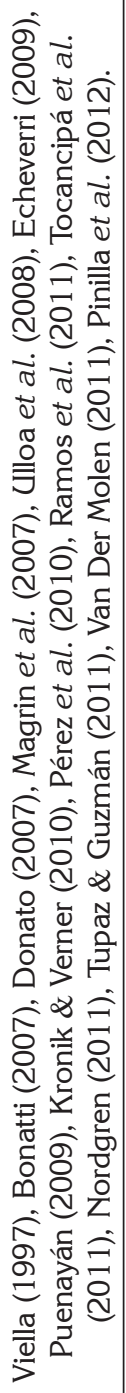 & 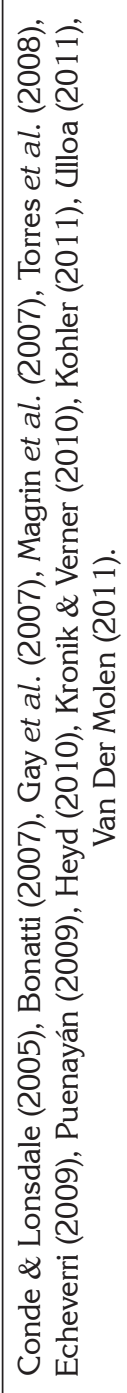 & 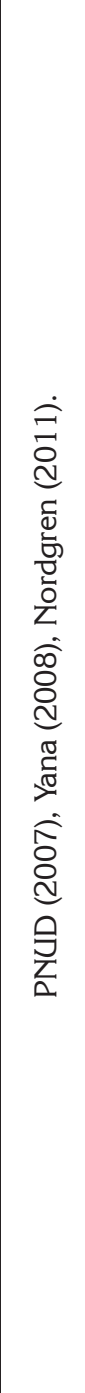 & 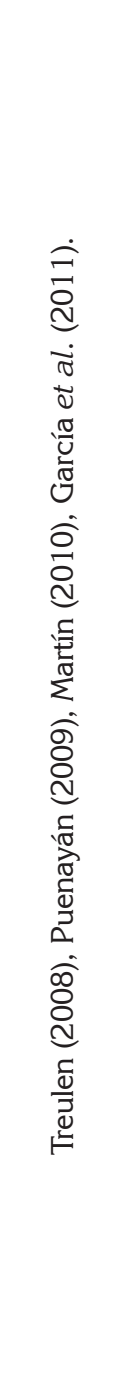 & 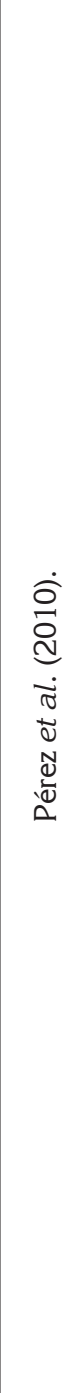 & 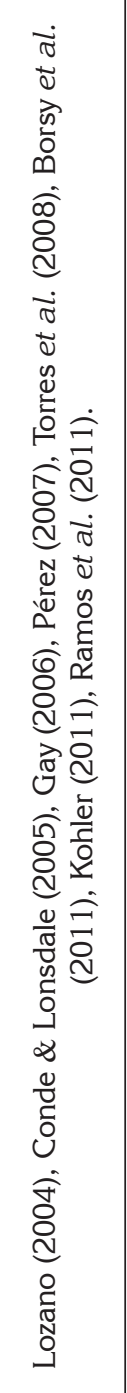 & 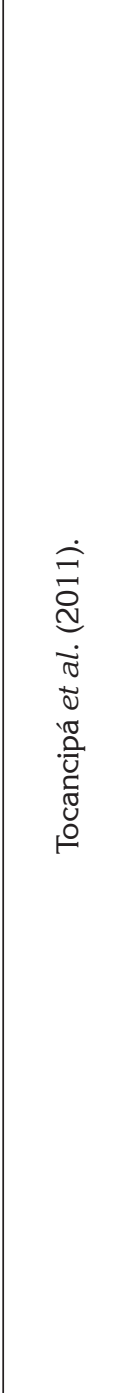 & 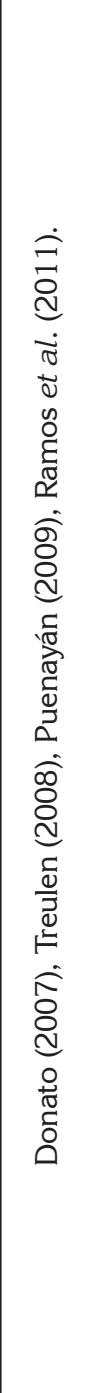 & 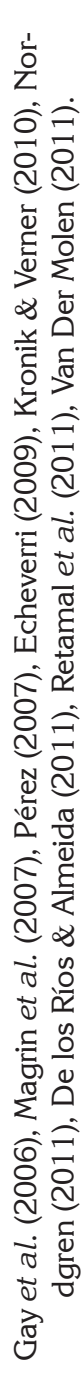 & 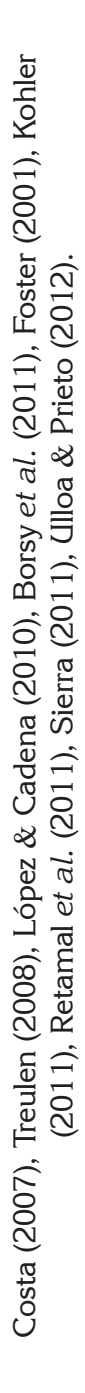 & 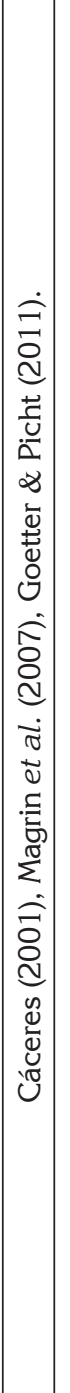 & 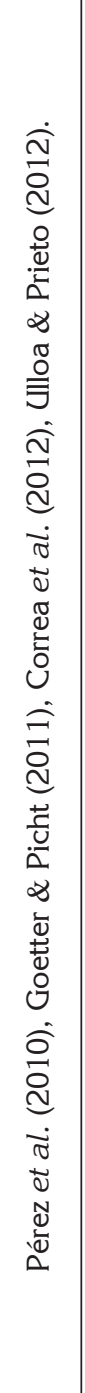 & 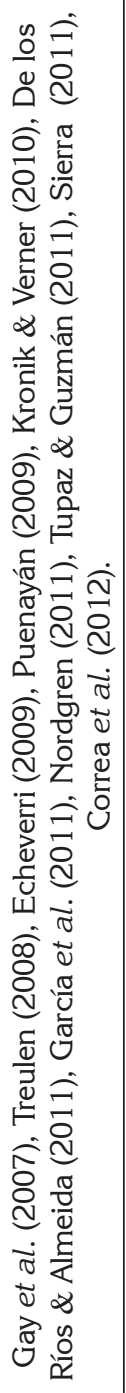 & 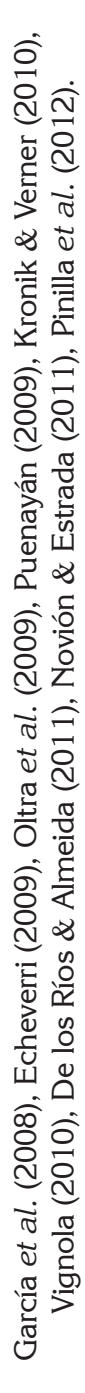 & 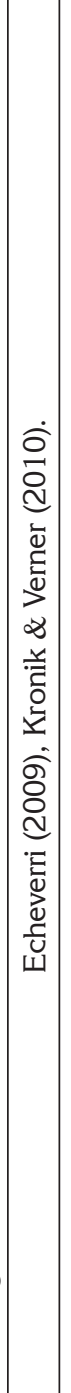 & 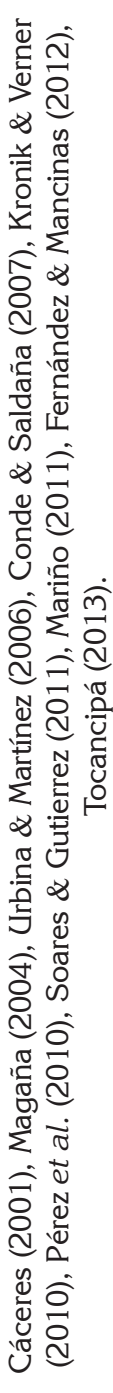 \\
\hline 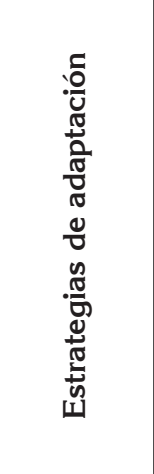 & 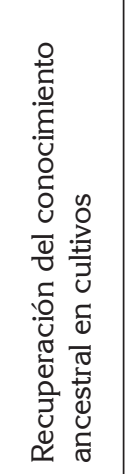 & 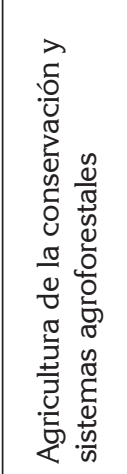 & 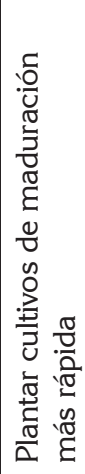 & 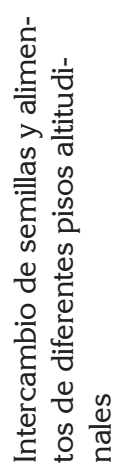 & 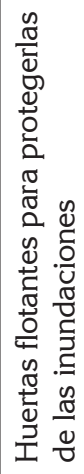 & 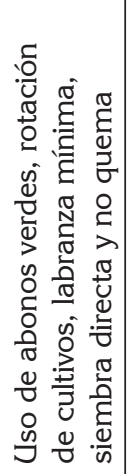 & 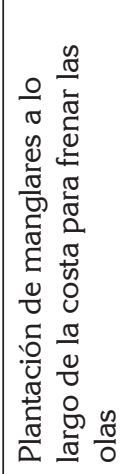 & 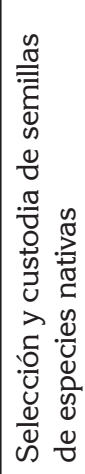 & 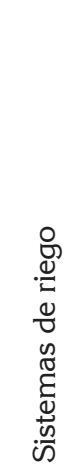 & 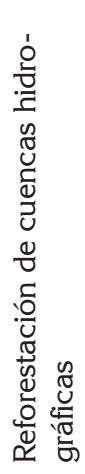 & 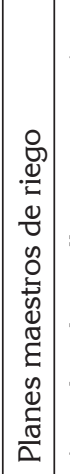 & 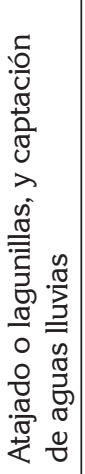 & 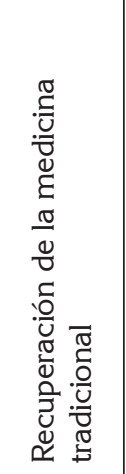 & 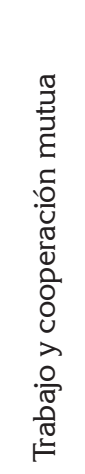 & 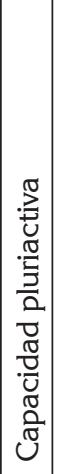 & 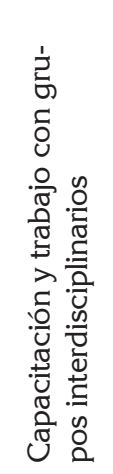 \\
\hline 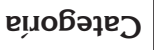 & \multicolumn{8}{|c|}{ e!̣enวədoıby } & \multicolumn{4}{|c|}{ еग!црІНH } & \multicolumn{4}{|c|}{ е!̣eұ!unwoว } \\
\hline
\end{tabular}


agropecuaria (57,6\%), (ii) comunitaria $(48,1 \%)$ e (iii) hídrica (40,4\%); lo anterior, a partir de los documentos detectados durante la investigación bibliográfica. Finalmente, la presente investigación bibliográfica acerca de las metodologías, herramientas de trabajo, percepciones y estrategias de adaptación al cambio climático responde al objetivo de involucrar a las comunidades locales campesinas e indígenas en la toma de decisiones, a nivel regional y nacional. De esta manera, se pretende ampliar el conocimiento y evitar deficiencias con respecto al desarrollo de políticas de mitigación y adaptación al cambio climático, por parte de los organismos públicos y privados interesados en atender este fenómeno.

Conflictos de intereses: El manuscrito fue preparado y revisado con la participación de todos los autores, quienes declaramos que no existe ningún conflicto de intereses que ponga en riesgo la validez de los resultados presentados. $\underline{\mathrm{Fi}-}$ nanciación: Este estudio fue financiado por los Grupos de Investigación para el Desarrollo Sostenible (INDESOS) e Ingeniería Ambiental (GIIAUD) de la Universidad Distrital F.J.C. (Colombia).

\section{BIBLIOGRAFÍA}

1. BONATTI, M. 2007. Cambios climáticos, percepciones humanas y desarrollo rural. Ed. Universidad de Buenos Aires. (Argentina). 114p.

2. BORSY, P.; GADEA, R.; VERA, E. 2011. Manejo forestal y agricultura de conservación. Experiencias de pequeños productores en la región oriental de Paraguay. Ed. Ministerio de Agricultura y Ganadería de Paraguay. (Paraguay). 232p.

3. CÁCERES, L. 2001. Vulnerabilidad, adaptación y mitigación al cambio climático en el Ecuador. Compendio de medidas, estrategias y perfiles de proyectos de los sectores energético, forestal, agrícola, marino costero y recursos hídricos. Ed. Min. Ambiente de Ecuador. 112p.

4. CAPEL, H. 1973. Percepción del medio y comportamiento geográfico. Rev. Geogr. (España). 7(1):58-150.

5. CONDE, C.; SALDAÑA, S. 2007. Cambio climático en América Latina y el Caribe: Impactos, vulnerabilidad y adaptación. Amb. Desarrollo. (Chile). 23(2):23-30.

6. CONDE, C.; LONSDALE, K. 2005. Engaging stake-holders in the adaptation process. En: Lim, B.; Spanger, E. (eds). Adaptation policy frameworks for climate change: Developing strategies, policies and measures. Ed. Cambridge University Press. (New York). p.47-66.
7. CORREA, S.L., TURBAY, S., VÉLEZ, M. 2012. Conocimiento ecológico local sobre ecosistemas marinos en dos comunidades costeras: El Valle y Sapzurro. Gest. Amb. (Colombia). 15(2):17-32.

8. COSTA, C. 2007. La adaptación al cambio climático en Colombia. Rev. Ing. (Colombia). 26(2):74-80.

9. DE LOS RÍOS, J.C.; ALMEIDA, J. 2011. Percepciones y formas de adaptación a riesgos socioambientales: análisis en contextos locales en la región del páramo de Sonsón, Antioquia, Colombia. Cuadernos Des. Rural. (Colombia). 7(65):107-124.

10. DONATO, L. 2007. Aportes de mujeres a los procesos de construcción de nuevas territorialidades indígenas. En: Donato, L.; Escobar, E.; Escobar, P.; Pazmiño, A.; Ulloa, A. (eds). Mujeres Indígenas, territorialidad y biodiversidad en el contexto latinoamericano. Ed. UNAL-Fundación Natura-UNODC. (Colombia). 303p.

11. DOUINIAS, E. 2011. Escuchando a los insectos: acercamiento entomológico al cambio climático entre pueblos indígenas africanos en bosques húmedos tropicales. En: Ulloa, A. (ed). Perspectivas culturales del clima. Ed. U.N. de Colombia. (Bogotá D.C.). p.223-246.

12. ECHEVERRI, J.A. 2009. Pueblos indígenas y cambio climático: el caso de la Amazonía colombiana. Bulletin de l'Institut Français d'Études Andines. (Perú). 38(1):13-28.

13. FERNÁNDEZ, R.; MANCINAS, R. 2012. Medios de comunicación y cambio climático. Ed. Fénix Editora. (España). 280p.

14. FOSTER, P., 2001. The potential negative impacts of global climate change on tropical montane cloud forests. Earth-Sci. Rev. (Netherlands). 55(1-2):73-106.

15. GARCÍA, C.; TENORIO, A.; MUÑOZ, F. 2011. Ciclos naturales, ciclos culturales: Percepción y conocimientos tradicionales de los Nasas frente al cambio climático en Toribio, Cauca, Colombia. En: Ulloa, A. (ed). Perspectivas culturales del clima. Ed. U.N. de Colombia. (Bogotá D.C.). p.247-274.

16. GARCÍA, L.; GARCÍA, M.; ANDRADE, F. 2008. La percepción social del cambio climático en la sierra de Huelva. Ed. U. de Huelva. (España). 1(20):227-236. 
17. GAY, C.; CONDE, C.; VINOCUR, M.; SEILER, R. 2007. Climatic treath spaces as a tool to assess current and future climate risks: Case studies in Mexico and Argentina. In: Leary, N.; Adejuwon, J.; Barros, V.; Burton, J. (eds). Climate change and vulnerability. Ed. Earthscan. (London). 432p.

18. GAY, C. 2006. Vulnerability and adaptation to climate variability and change: The case of farmers in Mexico and Argentina. Ed. The International START Secretariat (USA). 152p.

19. GAY, C.; ESTRADA, F.; CONDE, C.; EAKIN, H.; VILLERS, L. 2006. Potential impacts of climate change on agriculture: A case of study of coffee production in Veracruz, Mexico. Climatic Change. (USA). 79(3-4):259288.

20. GIFFORD, R.; STEG, L.; RESER, J. 2011. Environmental psychology. In: Martin, P.R.; Cheung, F.M.; Knowles, M.C.; Kyrios, K.; Littlefield, l.; Overmier, J.B.; Prieto, J.M. (eds). The handbook of applied psychology. Ed. Blackwell Publishing Ltd. (United Kingdom). 382p.

21. GOETTER, J.; PICHT, H.J. 2011. Adaptación al cambio climático: Cosecha de agua de lluvia con "Atajados" en Bolivia. Ed. Agencia de Cooperación AlemanaGTZ. (Bolivia). 5p.

22. HERNÁNDEZ, R. 1997. Metodología de la investigación. Ed. Mc Graw Hill. (México). 656p.

23. HEYD, T. 2010. Climate change, individual responsibilities and cultural frameworks. Hum. Ecol. Rev. (United States). 17(2):86-95.

24. HURTADO, J. 2000. Metodología de la investigación holística. Ed. SYPAL. (Venezuela). 666p.

25. KOHLER, A. 2011. Lecciones del "no-daño" en la tormenta tropical IDA. Impactos del proyecto RyGRAC en gestión del riesgo y en evaluación de "no-daño" en El Salvador. Ed. Kamar. (Salvador). 150p.

26. KRONIK, J.; VERNER, D. 2010a. Indigenous peoples and climate change in Latin America and the Caribbean. Ed. The World Bank (Washington DC). 208p.

27. KRONIK, J.; VERNER, D. 2010b. The role of indigenous knowledge in crafting adaptation and mitigation strategies for climate change in Latin America. In: Mearns, R.; Norton, A.(eds). Social dimensions of climate change: equity and vulnerability in a warming world. Ed. The World Bank (Washington DC). p.145-169.
28. LÓPEZ, C.; CADENA, J. 2010. Glaciares, nieves y hielos de América Latina. Cambio climático y amenazas. Ed. INGEOMINAS. (Colombia). 347p.

29. LOZANO, M. 2004. Evidencia de cambio climático: cambios en el paisaje. En: Martínez, J.; Fernández, A.; Osnaya, P. eds. Cambio climático: una visión desde México. Ed. Secretaría del Medio Ambiente y Recursos Naturales. (México). p.65-76.

30. MAGAÑA, V. 2004. El cambio climático global: comprender el problema. En: Martínez, J.; Fernández, A.; Osnaya, P. (eds). Cambio climático: una visión desde México. Ed. Secretaría del Medio Ambiente y Recursos Naturales. (México). p.17-28.

31. MAGRIN, G.; GAY, C.; GARCÍA, D.; CRUZ, J.C.; JIMÉNEZ, A.R.; MORENO, G.J.; NAGY, C.; VILLAMIZAR, A. 2007. Latin America Climate Change 2007: Impacts, Adaptation and Vulnerability. In: Parry, M.L.; Canziani, O.F.; Palutikof, J.P.; Van Der Linden, P.J.; Hanson., C.E. (eds). Contribution of Working Group II to the Fourth Assessment Report of the Intergovernmental Panel on Climate Change. Ed. Cambridge University Press. (United Kingdom). p.581-615.

32. MARIÑO, N. 2011. Reflexiones sobre la perspectiva cultural en las políticas de cambio climático en Colombia: un acercamiento al análisis cultural y espacial de las políticas públicas. En: Ulloa, A. (ed). Perspectivas culturales del clima. Ed. U.N. de Colombia. (Bogotá D.C.). p.495-528.

33. MARTíN, P. 2010. El trueque del cambio climático. Programa de Cambio Climático y Energía. Ed. PNUD. (Bogotá D.C.). 79p.

34. NORDGREN, M. 2011. Percepciones y síntomas de alteraciones en el clima de cuatro regiones de Bolivia y algunas oportunidades de resistencia al cambio climático. Ed. CIPCA. (Bolivia). 149p.

35. NOVIÓN, C.A.; ESTRADA, C. 2011. Percepción de los efectos vivenciales del cambio climático en una muestra de habitantes urbanos australes. Magallania. (Chile). 39(1):93-102.

36. O'CONNOR, R.; BORD, R.; FISHER, A. 1999. Risk perceptions, general environmental beliefs, and willingness to address climate change. Risk Anal. (USA). 19(3):461-471. 
37. OLTRA, C.; SOLÀ, R.; SALA, R.; PRADES, A.; GAMERO, N. 2009. Cambio climático: percepciones y discursos públicos. Rev. Cienc. Soc. (España). 2(1):1-23.

38. PABÓN, J.D. 2003. El cambio climático global y su manifestación en Colombia. Cuadernos Geog. (Colombia). 12(1-2):111-119.

39. PÉREZ, A.F. 2007. Mujeres indígenas de Guatemala y sus percepciones sobre el cambio climático. En: Ulloa, A.; Escobar, E. (eds). Mujeres indígenas y cambio climático. Perspectivas latinoamericanas. Ed. UNALFundación Natura-UNODC. (Bogotá D.C.). p.61-66.

40. PÉREZ, C. NICKLIN, C. DANGLES, O. VANEK, S. HALLOY, S. GARRETT, K. FORBES, G. 2010. Climate change in the high Andes: Implications and adaptation strategies for small-scale farmers. Int. J. Environ. Cult. Econ. Soc. Sustain. (Australia). 6(5):71-88.

41. PINILLA, M.C.; RUEDA, A.; PINZÓN, C.; SÁNCHEZ, J. 2012. Percepciones sobre los fenómenos de variabilidad climática y cambio climático entre campesinos del centro de Santander, Colombia. Amb. Des. (Colombia). 16(31):25-37.

42. PNUD. 2007. Colombia rural, Razones para la esperanza. Informe Nacional de Desarrollo Humano. Ed. Programa de las Naciones Unidas para el Desarrollo. (Colombia). 446p.

43. PUENAYÁN, Z. 2009. Percepción del cambio climático para los pastos del resguardo Panán, Nariño, Colombia. En: Pazmino, A.; Donato, L.M.; Escobar, E.; Escobar, P. (eds). Mujeres indígenas, territorialidad y biodiversidad en el contexto latinoamericano. Ed. U.N. de Colombia. (Bogotá D.C.). p.275-315.

44. RAMOS, C.; TENORIO, A.; MUÑOZ, F. 2011. Ciclos naturales, ciclos culturales: Percepción y conocimientos tradicionales de los Nasas frente al cambio climático en Toribio, Cauca, Colombia. En: Ulloa, A. (ed). Perspectivas culturales del clima. Ed. U.N. de Colombia. (Bogotá D.C.). p.247-274.

45. RETAMAL, R.; ROJAS, J.; PARRA, O. 2011. Percepción al cambio climático y a la gestión del agua: aportes de las estrategias metodológicas cualitativas para su comprensión. Amb. Soc. (Brasil). 14(1):175-194.

46. SIERRA, E. 2011. Las lluvias, el arcoíris y el trueno: representaciones simbólicas del paisaje y el sentido de lugar de los pueblos Kággaba, u'wa y misak, Colombia. En: Rocha, M. (ed). Antología de las literaturas indígenas de los Andes y la Sierra Nevada de Santa Marta. Ed. Ministerio de Cultura. (Colombia). 380p.

47. SOARES, D.; GUTIÉRREZ, I. 2011. Vulnerabilidad social, institucionalidad y percepciones sobre el cambio climático: un acercamiento al municipio de San Felipe, Costa de Yucatán. Ciencia Ergo. Sum. (México). 18(3):249-263.

48. STERN, P. 2000. Toward a coherent theory of environmentally significant behavior. J. Soc. Issues. (USA). 56(3):407-424.

49. TOCANCIPÁ, J.; ROSERO, J.; RESTREPO, F. 2011. Percepciones, representaciones religiosas y conocimiento local sobre el clima y sus cambios en el Pacífico Caucano. En: Ulloa, A. (ed). Perspectivas culturales del clima. Ed. U.N. de Colombia. (Bogotá D.C.). p.395-426.

50. TOCANCIPÁ, J. 2013. Replanteando el desarrollo: modernidad indígena e imaginación moral de David D. Gow. Antípoda. (Colombia). 16(1):239-248.

51. TORRES, J.; TENORIO, A.; GÓMEZ, A. 2008. Agroforestería: una estrategia de adaptación al cambio climático. Ed. Soluciones Prácticas-ITDG. (Perú). 126p.

52. TREULEN, K. 2008. Análisis sobre el impacto de los cambios climáticos en la vida de las mujeres mapuche de la región de la Araucanía, Pueblo Mapuche. Chile. En: Ulloa, A.; Escobar, E.; Donato, L.; Escobar, P. (eds). Mujeres indígenas y cambio climático. Perspectivas latinoamericanas. Ed. UNAL-Fundación Natura-UNODC. 234p

53. TUPAZ, D.; GUZMÁN, N. 2011. Tiempo y clima en la visión andina del pueblo de los pastos, Colombia y Ecuador. En: Ulloa, A. (ed). Perspectivas culturales del clima. Ed. U.N. de Colombia. (Bogotá D.C.). p.315-328.

54. ULLOA, A. 2011. Construcciones culturales sobre clima. En: ULLOA, A. eds. Perspectivas culturales del clima. Ed. U.N. de Colombia. (Bogotá D.C.). p.3354.

55. ULLOA, A.; ESCOBAR, E.; DONATO, L.; ESCOBAR, P. 2008. Mujeres indígenas y cambio climático. Perspectivas latinoamericanas. Ed. UNAL-Fundación Natura-UNODC. (Bogotá D.C.). 234p.

56. ULLOA, A; PRIETO, A. 2012. Pobladores urbanos y el clima. Flora Capital. (Colombia). 12(1):10-12. 
57. URBINA, J.; MARTÍNEZ, J. 2006. Más allá del cambio climático. Dimensiones psicosociales del cambio ambiental global. Ed. Universidad Nacional Autónoma de México. (México). 287p.

58. UZZELL, D. 2008. Challenging assumptions in the psychology of climate change. Psych. Bull. Australian Psycholog. Soc. 30(4):10-13.

59. VAN DER MOLEN, K. 2011. Percepciones de cambio climático y estrategias de adaptación en las comunidades agrícolas de Cotacachi, Ecuador. Ecuador Debate. (Ecuador). 82(1):145-158.

60. VIGNOLA, R. 2010. Estudio de la percepción y actitudes de la población costarricense sobre cambio climático. Ed. CATIE. (Costa Rica). 64p.
61. VIELLA, S. 1997. Vientos, lluvias, arcoíris: Simbolización de los elementos naturales en el ritual agrícola de la montaña Guerrero, México. En: Goloubinoff, M.; Katz, E.; Lammel, A. (eds). Antropología del clima en el mundo hispanoamericano. Ed. Abya-Yala. (Ecuador). p.225-236.

62. YANA, O. 2008. Diferencias de género en las percepciones sobre indicadores climáticos y el impacto de riesgos climáticos en el altiplano boliviano: estudio de caso en los municipios de Umala y Ancoraimes, departamento de La Paz. En: Ulloa, A.; Escobar, E.; Donato, L.; Escobar, P. (eds). Mujeres indígenas y cambio climático. Ed. U.N.I de Colombia. (Bogotá D.C.). p.43-55.

Recibido: Agosto 15 de 2013

Aceptado: Marzo 10 de 2014

\section{Como citar:}

Forero, E.L.; Hernández, Y.T.; Zafra, C.A. 2014. Percepción latinoamericana de cambio climático: metodologías, herramientas y estrategias de adaptación en comunidades locales. Una revisión. Rev. U.D.C.A Act. \& Div. Cient. 17(1): 73-85. 
